\title{
“¿ES NORMAL QUE SEA UN POCO DIFÍCIL DE LEER LA CONSIGNA?" LA ATENUACIÓN EN LAS PETICIONES DE HABLANTES NO NATIVOS DE ESPAÑOL
}

\section{"IS IT NORMAL THAT THE INSTRUCTIONS ARE A LITTLE DIFFICULT TO READ?" MITIGATION IN REQUESTS BY NON-NATIVE SPEAKERS OF SPANISH}

\author{
AurÉlie MARSILY ${ }^{1}$ \\ Université catholique de Louvain \\ aurelie.marsily@uclouvain.be
}

Recibido: 23/12/2016

Aceptado: 09/03/2017

\begin{abstract}
Resumen
Este artículo analiza la producción de peticiones en español por parte de estudiantes francófonos de Bélgica. Más específicamente, estudia los diferentes recursos que usan para atenuar sus peticiones, a saber, la atenuación de lo dicho y del decir. Los datos han sido recopilados mediante interacciones naturalizadas, siguiendo una metodología inicialmente desarrollada por Tran (2006). Los resultados del análisis de las peticiones se analizan mediante una taxonomía elaborada a partir del estado de la cuestión y de un análisis de corpus. Estos resultados se alejan en parte de lo que se suele encontrar en estudios anteriores: encontramos muchas peticiones indirectas o peticiones reorientadas hacia el emisor. En cuanto al análisis de la atenuación,
\end{abstract}

\begin{abstract}
This article analyses the production of requests in Spanish by French-speaking students in Belgium. More specifically, it studies the different strategies that they use to mitigate their requests, namely atenuación del decir and atenuación de lo dicho. The data were collected through naturalized interactions, following Tran's (2006) initial methodology. The results of the analysis of those requests are analysed by means of a previously elaborated taxonomy, based on the literature and on a corpus analysis. Those results differ partly from what is traditionally found in the literature: a huge amount of indirect requests were found, as well as reoriented requests (towards the speaker). As for the analysis of mitigation, the students
\end{abstract}

1 Agradecemos los valiosos comentarios de Barbara De Cock (Université catholique de Louvain), Pedro Gras (Universiteit Antwerpen) y de los revisores anónimos sobre versiones anteriores de este trabajo.

Para citar este artículo / To cite this article: Marsily, Aurélie (2018): “¿Es normal que sea un poco difícil de leer la consigna?" La atenuación en las peticiones de hablantes no nativos de español. García Ramón, Amparo y Soler Bonafont, María Amparo (Eds.): ELUA: Estudios de atenuación en el discurso, Anexo IV, págs. 251-268.

Enlace / Link: http://dx.doi.org/10.14198/ELUA2018.Anexo4.14 
se hace uso principalmente de la atenuación del decir. Además, se puede afirmar que las peticiones indirectas ya son una forma de atenuar la petición, en vez de optar por estrategias más directas. Otro recurso de atenuación es optar por no realizar la petición o hacer uso de gestos y de miradas para no formularla de manera verbal.

PALABRAS CLAVE: español como lengua extranjera, petición, atenuación, pragmática, lenguaje oral. mainly use atenuación del decir. Moreover, it can be stated that the indirect requests are already a way to mitigate the requests, instead of opting for more direct strategies. Another means to mitigate a request is choosing not to perform the request or using gestures and looks in order not to produce it verbally.

KEY WORDS: Spanish as a second language, request, mitigation, pragmatics, oral speech.

\section{INTRODUCCIÓN Y OBJETIVOS}

La formulación de peticiones constituye una acción cotidiana, sea cual sea el ámbito en el que estén formuladas. Visto el carácter muy frecuente de este acto de habla, es interesante analizar cómo se suelen caracterizar. Nos centramos en las peticiones expresadas en el ámbito universitario y, más precisamente, por parte de los estudiantes hacia sus profesores. $\mathrm{Si}$ bien un número considerable de estudios contrastivos se han focalizado en la formulación de peticiones, entre otros el Cross Cultural Speech Act Research Project (a continuación, CCSARP $^{2}$ ), no se ha llevado a cabo ningún estudio que compare las peticiones en francés y en español en el contexto académico ${ }^{3}$. En este artículo, proponemos centrarnos particularmente en la manera en que los estudiantes francófonos de Bélgica expresan sus peticiones en español. En futuros estudios, estos resultados se confrontarán con el análisis de peticiones formuladas por estudiantes nativos de español, y por estudiantes nativos de francés.

La atenuación de los actos de habla juega un papel muy importante en el aprendizaje de una lengua extranjera, no sólo porque permite mitigar el mensaje sino también porque refleja el grado de dominio de esta lengua. Efectivamente, como varios autores ya han comentado (Janney Arndt 1992; Escandell Vidal 1996, 2009; Alonso Pérez-Ávila 2005; Valero Aguilar 2008), comunicarse de manera eficaz en una lengua "no consiste sólo en conocer y manejar bien su gramática, sino que implica también hacerse con un dominio adecuado de un complejo conjunto de conocimientos de naturaleza extragramatical" (Escandell Vidal 2009: 98). La atenuación es, por ello, un recurso útil a la hora de matizar nuestros propósitos. Se define comúnmente como "una categoría pragmalingüística cuya función principal consiste en minimizar la fuerza ilocutiva de los actos de habla y el papel de los participantes de la enunciación con el fin de lograr la meta prevista, el acuerdo (o en aminorar en su caso el desacuerdo), que es el fin último o primero, según se mire, de toda conversación". (Briz 2007: 12). Como señalan Albelda y Briz (2010), la atenuación es un recurso lingüístico. Además, es "una estrategia de distancia lingüística y de acercamiento social" (Briz 2007: 37). Según el mismo autor (Briz 2007: 17), "la presencia mayor o menor de atenuantes en una conversación no supone necesariamente mayor o menor cortesía", ya que, como ha probado en su estudio, son dos conceptos entre los cua-

2 Este proyecto (Blum-Kulka et alii 1989) investiga la realización de peticiones y disculpas en ocho lenguas diferentes.

3 No obstante, sí se encuentran estudios en otros ámbitos, por ejemplo, Castillo Lluch (2009), quien analiza conversaciones telefónicas de la RENFE y de la SNCF. 
les no siempre hay correlación. Por otra parte, existen dos tipos de atenuación, a saber, la atenuación de lo dicho y la atenuación del decir (Albelda (2010), basadas en Briz (1998)). La atenuación del decir se relaciona típicamente con la fuerza ilocutiva de un acto de habla, mientras la atenuación de lo dicho "se refiere al contenido proposicional, en la que se atenúa también indirectamente el decir: es aquella por la que se minimiza la cantidad o se expresa un elemento de la proposición de forma vaga o imprecisa. Esto se consigue principalmente con recursos morfológicos o mediante la selección léxica". (Albelda 2010: 3). Teniendo en cuenta estas definiciones, consideramos que una petición que se expresa por medio de una estrategia indirecta constituye una manera de atenuar la petición que entra en la categoría de la atenuación del decir. Para analizar los datos, nos basamos en las obras de Briz (2007), quien estudia la atenuación en conversaciones informales y entrevistas, y en la propuesta metodológica de Briz y Albelda (2013) y de Albelda y Cestero (2011), quienes han pretendido "revisar, aunar y completar las clasificaciones existentes" (2011: 13). Se puede apreciar la clasificación de estas autoras en la parte analítica y la acompañan ejemplos explicativos de cada uno de los tipos de atenuación. A continuación, discutimos el estado de la cuestión acerca de las peticiones en español y establecemos las preguntas de investigación y las hipótesis. Después de presentar la metodología utilizada para llevar a cabo el análisis (Sección 2), nos enfocamos en el análisis de la formulación de las peticiones y de su atenuación (Sección 3). En la última sección (Sección 4), presentamos nuestras conclusiones.

De la bibliografía sobre las peticiones en español (nativo) surgen varios resultados. En estudios sobre prestaciones de un servicio en Madrid (Placencia 2005) y en Sevilla (Placencia y Mancera Rueda 2011), las autoras concluyen que las peticiones se hacen mediante una estrategia directa y con muy pocos mitigadores (Bataller 2013). Estos estudios confirman lo que estudios anteriores ya habían destacado, a saber que en español peninsular, no se usan muy frecuentemente peticiones indirectas. En su estudio de 2010, Bataller destaca la preferencia en español por utilizar estrategias indirectas convencionales que son las preguntas preparatorias de voluntad, de habilidad ${ }^{4}$ y los interrogativos simples ${ }^{5}$ (ver taxonomía en Anejo 1). Otros estudios muestran que tanto las peticiones orientadas hacia el oyente como las elípticas son herramientas utilizadas de manera frecuente por los hablantes nativos del español peninsular en contextos transaccionales (Mir 1993, Placencia 1998).

No obstante, estas consideraciones conciernen el habla de los nativos del español. Bien es cierto que un estudiante no nativo de una lengua meta no siempre tiene el mismo comportamiento lingüístico que el hablante nativo. Este es debido al hecho que cualquier aprendiente estudia una lengua extranjera con un bagaje cultural y lingüístico propios de su lengua materna y de las demás lenguas que conozca. Sin embargo, a este respecto, Escandell Vidal (1996) dice que un aprendiz no puede simplemente transferir los conocimientos de su lengua materna a la lengua extranjera.

Teniendo en cuenta las aportaciones anteriores, formulamos tres hipótesis. La primera concierne el número de peticiones (no) formuladas. Como explicamos en la Sección 2, cree-

4 Blum-Kulka et alii (1989: 280) definen las preguntas preparatorias como peticiones en las que "the utterance contains reference to a preparatory condition for the feasability of the Request, typically one of ability, wilingness, or possibility, as conventionalized in the given language".

5 Esta categoría ha sido explicada por Carduner (1998) como una pregunta que refleja la no evidencia de conformidad (nonobviousness of compliance). 
mos que las peticiones necesarias para poder llevar a cabo la tarea (pedir un bolígrafo, pedir una hoja donde se pueda leer mejor las consignas), serán más formuladas que las peticiones menos necesarias o no directamente relacionadas con la tarea. Se trata de las peticiones para comer o beber algo, pero también las de pedir un diccionario o un borrador. Suponemos que estas peticiones dependen del nivel de lengua de los participantes. Segundo, esperamos un mayor uso de atenuación en las peticiones que constituyen un mayor grado de amenaza hacia la imagen del destinatario ${ }^{6}$, con el fin de matizar y debilitar el impacto impositivo sobre el oyente. Tercero, visto el tipo de situación comunicativa, postulamos que hay más atenuación del decir que de lo dicho en el corpus. Efectivamente, visto que el objeto de las peticiones que tienen que formular los estudiantes no implica una amenaza muy fuerte de la imagen de los participantes, pensamos que no es imprescindible que mitiguen el contenido proposicional de sus peticiones, es decir, que utilicen mucha atenuación de lo dicho. Estas hipótesis serán comprobadas en la Sección 3, a la luz de nuestros datos.

\section{METODOLOGÍA}

Este estudio y la recolección de los datos se fundan en la metodología del juego de rol naturalizado (Naturalized Role Play) de Tran (2006). Como su nombre indica, los informantes siguen un guion preestablecido por el autor. Está diseñado para recopilar habla producida en un contexto espontáneo ${ }^{7}$ y auténtico. El concepto naturalizado gira en torno a una idea clave: distraer el participante del foco de estudio. Tran concibió este juego de rol naturalizado para recoger segundos turnos de habla (respuestas a cumplidos, que el entrevistador introducía a lo largo del juego de rol, sin el conocimiento previo del participante). A fin de utilizar este método para el presente estudio, traspusimos la idea para primeros turnos de habla, es decir para peticiones. Nos alejamos de la denominación de juego de rol, ya que los estudiantes no siguen ningún juego de rol. Efectivamente, en el caso de este estudio, los estudiantes se encuentran en su propia posición (contrariamente a la entrevistadora, que sigue un guion minucioso ${ }^{8}$ ) y están enfrentados con situaciones con las que se pueden identificar fácilmente. Veamos un ejemplo del tipo de petición que se puede obtener con el método que hemos desarrollado. La entrevistadora crea un entorno en el que falta un bolígrafo, a fin de elicitar la petición. En (1), el participante nota la ausencia de bolígrafo para rellenar el formulario y mira primero a la entrevistadora antes de hablar. Como ella tiene cuidado de no mirar al participante para obligarlo a formular la petición verbalmente, el informante se decide a hablar y responde a la situación comunicativa mediante una "petición" indirecta (cf. Sección 3).

6 Según Brown y Levinson (1987), las peticiones son, por definición, actos amenazadores de la imagen (face threatening act) de los interlocutores, ya que son intrínsicamente impositivas. Además, "el modelo de Brown y Levinson viene a incidir en que el peso de tres factores -el del poder relativo, el de la distancia social, y el del grado de imposición- permite al emisor calcular la repercusión del acto de amenaza a la imagen y seleccionar el nivel de cortesía requerido para mitigar el riesgo potencial" (Curcó (1998: 133).

7 Según Beebe y Cummings (1996), el habla es espontáneo cuando los participantes no son conscientes de ser observados o estudiados. En nuestra aceptación de la definición, es espontáneo cuando los participantes no son conscientes del foco de atención del estudio. En nuestro estudio, los estudiantes creen que vienen para una expresión escrita pero les grabamos para recopilar peticiones.

8 Por este motivo, tan solo las intervenciones de los participantes han sido analizadas, no las de la investigadora. 
(1) $<$ MF001 $>$ Voy a tomar un... (ruido metálico de silla)

$<$ PROFE $>$ ¿Sí? $<$ MF001 $>$ Porque no tengo uno

$<$ PROFE $>$ Ah, no, ¿quieres uno? Aquí... aquí

$<$ MF001 $>$ Ah gracias. (tos)

(IN_001_MF)

A continuación, presentamos el procedimiento metodológico para nuestra investigación (2.1) y los participantes incluidos en el estudio (2.2), y luego describimos brevemente los principales criterios de la taxonomía (2.3).

\subsection{Procedimientos de recopilación de datos}

Los estudiantes fueron grabados con vídeo y grabadora de voz durante toda la entrevista, constituyendo así un corpus oral de peticiones en interacciones naturalizadas en español (COPINE). La entrevista se divide en cuatro partes, que se pueden apreciar en la Figura 1.

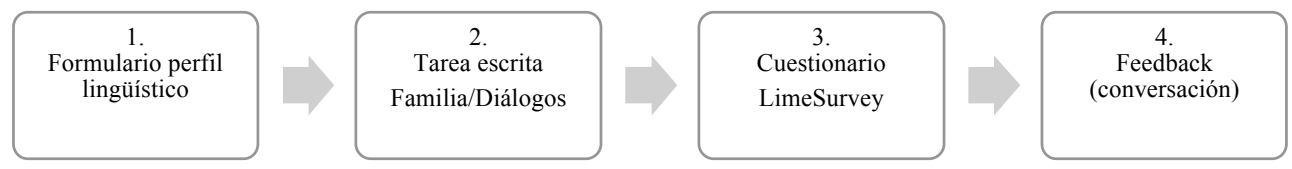

Figura 1. Diseño de la entrevista.

La primera parte consiste en un formulario sobre el perfil lingüístico de los participantes, que sirve para validar su selección 9 . La segunda parte es la tarea escrita. Fue pensada como distractor, para elicitar peticiones por parte del participante. Un ejemplo de petición, lo constituye una hoja mala donde no se pueden leer las instrucciones, que incita el estudiante a pedir una hoja de mejor calidad. En la tercera parte del experimento, se trata de completar un formulario en línea sobre sus percepciones en torno a la cortesía y la adecuación de algunas peticiones en contextos académicos, comparables con las situaciones de la tarea escrita. Finalmente, la última parte consta de algunas preguntas sobre sus impresiones a lo largo del experimento. Es una etapa en que pueden surgir comentarios importantes en cuanto a ciertas producciones de los estudiantes.

Se les había informado con antelación a los estudiantes que participarían en una redacción sobre un tema preciso. Al llegar a la sala de prueba, estaban convencidos de que la redacción era el centro de atención de la investigación. No obstante, durante la realización de esta tarea, se les ponían dificultades o necesidades cuyo objetivo era generar la producción de peticiones. Algunos ejemplos de estas necesidades son la falta de bolígrafo para rellenar el formulario, la distribución de una fotocopia de calidad insuficiente como para poder realizar la tarea de manera óptima, la mala pronunciación de una pregunta que les impedía la comprensión, etc. De esta forma, las peticiones formuladas son auténticas, ya que los estudiantes no están al tanto de este foco de atención.

9 Los estudiantes que tenían una lengua materna diferente del francés fueron descartados del estudio. Asimismo, fueron excluidos los estudiantes no belgas (cf. Sección 2.2). 


\subsection{Participantes}

Los participantes son todos estudiantes universitarios de lengua española (filología o traducción/interpretación) en los últimos años de estudio (último año de grado o de máster). Todos son belgas y tienen el francés como lengua materna ${ }^{10}$. De los 29 informantes, la mayoría son mujeres (26) y tres son varones (véase la Tabla 1). Esta repartición poco equilibrada refleja la realidad de los estudios de filología en Bélgica. Sin embargo, en etapas ulteriores, nuestro objetivo es equilibrar más el grupo de participantes. Todos los experimentos se hacen con la autora del presente artículo, es decir, una profesora ayudante no nativa de español, cuya lengua materna es el francés. Aunque su nivel de lengua y su género podrían jugar un papel en los resultados de los estudiantes, estas dos variables no constituirán nuestro enfoque.

\begin{tabular}{|l|c|}
\hline Mujeres & 26 \\
\hline Hombres & 3 \\
\hline Total & $\mathbf{2 9}$ \\
\hline
\end{tabular}

Tabla 1. Repartición de hombres y mujeres francófonos.

\subsection{Taxonomía}

El marco de referencia dentro del cual se realiza este estudio reúne las aportaciones de las investigaciones sobre las peticiones, entre las que figuran las desarrolladas por BlumKulka (1987) y más tarde en el CCSARP de Blum-Kulka et alii (1989), así como por Carduner (1998), Hassall (2003) y Bataller (2010). Para una explicación detallada sobre la taxonomía en la que basamos nuestro estudio, remitimos a Marsily (2018). Además de basarse en la literatura, la primera versión de la taxonomía fue elaborada tras un análisis de dos corpus orales: el CORLEC para la lengua española, y el CIEL-F para el francés. Añadimos elementos a la taxonomía tras este análisis ( $c f$. Sección 3), ya que la taxonomía inicial no permitía dar cabido a una parte de los datos del análisis. Blum-Kulka (1987) distingue entre las peticiones directas, las indirectas convencionales y las indirectas no-convencionales. Añadimos la cuarta categoría de la Tabla 2, que explicamos a continuación.

Esta repartición representa una escala de tipos mutuamente exclusivos de estrategias de petición. Esta noción de (in)dirección se basa en el grado inferencial que se necesita para llegar a interpretar la fuerza ilocutiva del enunciado (Blum-Kulka 1987: 133). La primera categoría (las peticiones directas) abarca las peticiones que incluyen verbos performativos, modos derivables como el imperativo, y declaraciones de obligación o de volición. Las peticiones indirectas convencionales, que forman la segunda categoría, se definen por contener dos actos de habla que se realizan simultáneamente. Están formadas por fórmulas de sugerencias, preguntas preparatorias (sobre la capacidad, la voluntad, etc.) y el interrogativo simple (típicamente con el indicativo y bajo pregunta). En la tercera categoría se encuentran las peticiones indirectas no convencionales, que son formulaciones donde no se puede derivar directamente la intención ilocutiva. La petición solo puede ser interpretada

10 Una estudiante nació en Luxemburgo, pero su familia es belga (ella incluida) y su lengua materna es el francés. 


\begin{tabular}{|c|c|}
\hline Petición & Ejemplo \\
\hline \multicolumn{2}{|l|}{ Convencional directa } \\
\hline Modo derivable & Cierra la puerta. \\
\hline Performativos & Te pido cerrar la puerta. \\
\hline Declaración obligativa & Tienes que cerrar la puerta. \\
\hline Declaración volitiva & Quiero que cierres la puerta. \\
\hline \multicolumn{2}{|l|}{ Convencional indirecta } \\
\hline Fórmulas de sugerencia & ¿Por qué no cierras la puerta? \\
\hline Pregunta preparatoria & ¿Puedes cerrar la puerta? \\
\hline Interrogativo simple & ¿Cierras la puerta? \\
\hline \multicolumn{2}{|l|}{ No convencional indirecta } \\
\hline Alusión & Hace frío aquí. \\
\hline Elipsis & ¡La puerta! \\
\hline \multicolumn{2}{|c|}{ Reorientación de la petición hacia el emisor } \\
\hline Permiso & ¿Puedo cerrar la puerta? \\
\hline Pregunta (sobre desarrollo) & ¿Hace falta cerrar la puerta o algo? \\
\hline Aserción & Voy a cerrar la puerta. \\
\hline
\end{tabular}

Tabla 2. Taxonomía de las peticiones.

tomando en cuenta el contexto. Blum-Kulka y House (1989) diferencian entre las alusiones firmes (strong hints) y las leves (mild hints). Preferimos discernir las alusiones bajo forma de preguntas frente a las asertivas (introducidas por Hassall 2003) ${ }^{11}$, como en (2), donde el estudiante menciona que no puede leer la copia, sugiriendo, con esta alusión, que la profesora le dé una copia de mejor calidad. Las elipsis son peticiones interesantes en el ámbito de nuestro experimento ya que son bastante frecuentes también. Se definen por la elipsis del verbo, pero se clasifican según los elementos de la petición que se mantienen. Discernimos tres tipos de elipsis: el objeto de la petición (un poco de agua en (3)), la persona que tiene que realizar la petición (es decir un vocativo - inexistente en nuestro corpus) o una fórmula gramaticalizada que es una expresión fija que puede encontrarse por sí sola y que, en contexto, sugiere una petición. Se ilustra en (4), donde el hablante pide que se repita la información con la interrogación ¿Cómo?

(2) $<$ FF006> En la copia no se ha... que no veo muy bien

$<$ PROFE $>$ ¿Qué es esto?

$<$ FF006 $>$ (risas)

$<$ PROFE $>$ Uy, lo siento, sí eso es...

$<$ FF006 $>$ (risas)

$<$ PROFE $>$ Te voy a dar otra.

(IN_006_FF)

11 Otros cambios se hicieron en comparación con las primeras taxonomías. Carduner (1998) introdujo el interrogativo simple, la forma elíptica y la diferenciación entre cuatro estrategias de preguntas preparatorias. Bataller (2003) integró los performativos explícitos e implícitos bajo una sola categoría (performativos). 
(3) $<$ PROFE $>$ OK. Pero si quieres algo de beber o de comer...

$<$ FF017 $>$ Euh... Un poco de agua.

$<$ PROFE $>$ Un poco de agua, sí, te lo hago.

$<$ FF017 $>$ No sabía que estaba por para nosotros.

$<$ PROFE $>$ No es para mí, eh ¿todo eso para mí? (risas)

(IN_017_FF)

(4) $<$ PROFE $>$ Ah sí, y ¿quieres (incomprensible)?

$<$ MF012> ¿Cómo?

$<$ PROFE $>$ Que si quieres algo eh bebidaaa eh

$<$ MF012 $>$ (risas)

$<$ PROFE $>$ No pero como vienes para ayudarme pues yo también...

$<$ MF012 $>$ (risas)

(IN_012_MF)

Como ya hemos mencionado, la clasificación de Blum-Kulka et alii (1989) no permitía dar cabida a una parte considerable de los datos del presente artículo. Además de las estrategias ya presentadas, surgen otras, las de la cuarta categoría. Efectivamente, en numerosos casos, el hablante, en vez de pedir al oyente que realizara una petición, se dirige hacia el oyente (a) para pedir el permiso de realizarla él mismo, (b) para hacer una pregunta que le permite realizar la petición él mismo o (c) para informar al oyente que la va a realizar él mismo. Estas estrategias forman las peticiones reorientadas hacia el emisor, que explicamos en la sección de resultados.

\section{RESULTADOS}

En este apartado, analizamos la formulación de peticiones utilizadas por los hablantes no nativos del español, que tienen como lengua materna el francés (de Bélgica). Estudiamos sus condiciones de uso y la manera en que están atenuadas. Siempre que sea oportuno, establecemos relaciones con la lengua nativa para comprender mejor la producción de los hablantes francófonos. Concretamente, ofrecemos un análisis de recursos atenuadores utilizados por los estudiantes francófonos en sus peticiones en español. Nos interesan los resultados que conciernen, por una parte, la realización o no de las peticiones previstas (Sección 3.1) y, por otra, la presencia vs. ausencia de atenuación en estas peticiones (Sección 3.2) También damos una visión de conjunto del contexto en que se tienden a atenuar las peticiones, así como del tipo de petición (desde el punto de vista gramatical) con el que se suele encontrar atenuación (Sección 3.3).

\subsection{Peticiones realizadas y no realizadas}

La metodología que se ha desarrollado para llevar a cabo esta investigación permite generar peticiones de manera auténtica y espontánea por parte de los participantes. No obstante, también conlleva una consecuencia para la recopilación de los datos. Aunque sometamos a los estudiantes a situaciones de necesidad o de dificultad, las peticiones que producen dependen plenamente de sus propias necesidades o dificultades. En el caso de un estudiante que llega al experimento con un bolígrafo en la mano, por ejemplo, la petición de un bolígrafo no es realizada. Pensamos en otro ejemplo, es decir, una frase que se pronuncia de manera incomprensible por parte de la profesora implicada en el experimento. Hubo 
casos en los que el participante actuaba como si hubiera entendido la frase ininteligible. En otros casos, se notaba por el lenguaje no verbal (las miradas) que el estudiante quería pedir que se repitiera la información pero que no se atrevía. En la Figura 2, se presenta la repartición de peticiones realizadas. El material obtenido recopila 102 peticiones. Las peticiones más formuladas son, respectivamente, la petición de una fotocopia de mejor calidad que la entregada al estudiante (28\%), la reformulación de una frase incomprensible (22\%), el bolígrafo $(20 \%)$ y permiso para desplazar un bolso que está en la silla en la que tienen que sentarse (14\%). Como estas peticiones son etapas necesarias para el estudiante, es normal que representen un número importante de peticiones del corpus. Para realizar la tarea, el estudiante necesita tanto utilizar un bolígrafo, como poder leer las consignas en la fotocopia. Por consiguiente, nuestra primera hipótesis en cuanto a las peticiones formuladas se corrobora. Efectivamente, las peticiones necesarias, por las que el estudiante debe necesariamente pasar para cumplir la tarea, son las que más se formulan en la entrevista.

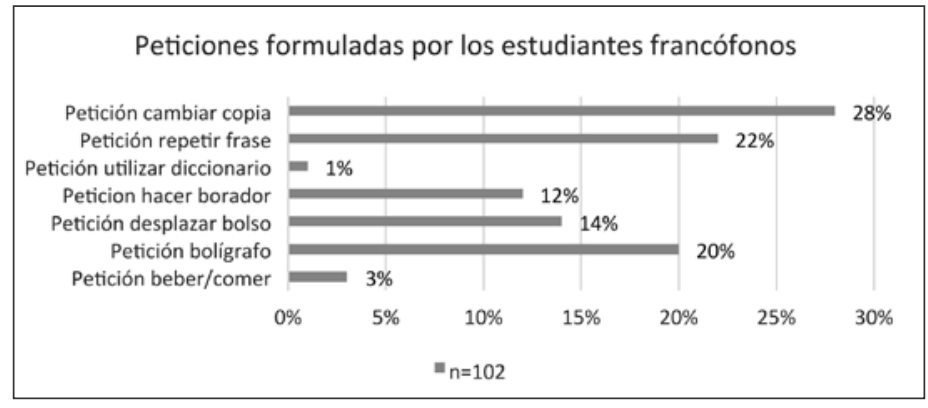

Figura 2. Peticiones formuladas por los estudiantes francófonos.

También encontramos peticiones formuladas de manera no verbal, es decir con gestos y miradas, especialmente en los casos de pedir la repetición de una información o de solicitar una fotocopia de mejor calidad. Estas peticiones no se han tenido en cuenta para este análisis de la atenuación, porque queremos ofrecer un análisis lingüístico de la atenuación.

El porcentaje de las peticiones no realizadas es bastante elevado. Esto significa que las peticiones que habíamos planeado no siempre ocurren. Las razones de esta no realización pueden ser múltiples. En algunas situaciones, los estudiantes pueden resolver el problema ellos mismos y así evitar la petición. Frente a una ausencia de bolígrafo para realizar la tarea escrita, varios estudiantes eligen ir a buscar uno en su bolso, en vez de pedírselo a la profesora. Además, pocos estudiantes aceptan la invitación para beber o comer algo, lo que está directamente relacionado con sus propias necesidades en el momento del experimento y/o con lo que les parece adecuado en este contexto. La situación de pedir un diccionario se explica de una manera similar. Visto que se les pide a los estudiantes imaginarse en el contexto de la petición, en el que no dispondrían del diccionario, la casi totalidad de los estudiantes completan la tarea sin hacer uso del diccionario. Luego, una última causa de la no realización de ciertas peticiones es que no se haya podido incluir la necesidad de la petición en el experimento o que no se haya logrado hacerlo bien. En algunas ocasiones, no se logró pronunciar la "frase incomprensible" lo suficientemente mal como para que el estudiante no lo entendiera. En otras, se olvidó algún elemento que impidió la formulación de la petición por parte del estudiante. 
No obstante, cabe añadir que, al igual que un estudiante omite producir ciertas peticiones por ausencia de necesidad, también puede formular otras. Estas peticiones incluyen, sin pretensiones de exhaustividad, pedir más información sobre la consigna, el permiso de empezar la tarea, información sobre la fecha del día, permiso para sentarse, etc. Sin embargo, visto el carácter heteróclito de estas peticiones y la dificultad que representan a nivel comparativo, las apartamos del análisis de este artículo.

La Tabla 3 incluye las estrategias utilizadas por los participantes del experimento. A continuación, explicamos la repartición de dichas estrategias.

\begin{tabular}{|c|c|}
\hline Estrategia & Frecuencia \\
\hline Convencional directa & $\mathbf{0}$ \\
\hline Convencional indirecta & 1 \\
\hline Pregunta preparatoria & 1 \\
\hline No convencional indirecta & 71 \\
\hline Alusión & 44 \\
\hline Elipsis & 27 \\
\hline $\begin{array}{l}\text { Reorientación de la petición hacia el } \\
\text { emisor }\end{array}$ & 30 \\
\hline Pregunta (sobre desarrollo) & 15 \\
\hline Permiso & 7 \\
\hline Aserción & 8 \\
\hline Total & 102 \\
\hline
\end{tabular}

Tabla 3. Repartición de estrategias de peticiones.

Por una parte, las peticiones directas son inexistentes en el corpus. En el caso de la petición convencional indirecta, se apunta tan solo una ocurrencia. Por otra parte, se observa que la estrategia indirecta no convencional es la más frecuente del corpus. Dentro de este grupo, la alusión es el recurso preferido de los aprendientes.

La última sección de la taxonomía recopila las formulaciones donde se hace una 'reorientación de la petición hacia el emisor'. En este corpus, se hallan muchas peticiones que se orientan más bien hacia el emisor (el estudiante) pero que incluyen al destinatario (la profesora). Algunas formulaciones ya no son peticiones sino más bien respuestas a situaciones comunicativas. Se destacan tres recursos diferentes. La primera categoría ( $c f$. ejemplo (5)), concierne las peticiones en forma de preguntas sobre el desarrollo del experimento y que llevan a una acción por parte del estudiante. En este caso, el estudiante pregunta si necesita tomar algo para escribir. Si la profesora hubiera contestado que sí, habría llevado al estudiante a tomar un instrumento para escribir. Aquí, surge una respuesta negativa puesto que la profesora lleva todo el material previsto. La segunda categoría agrupa las peticiones de permiso. En el ejemplo (6), el estudiante pide permiso para tomar un bolígrafo de su mochila. Aquí también, la profesora da una respuesta negativa. La última categoría son más bien aserciones que funcionan como peticiones, del mismo modo que las alusiones, pero se centran en la acción del hablante. En otros términos, son actos de habla asertivos con los que el estudiante informa de lo que va a hacer para que el destinatario le confirme que está de acuerdo. Como se puede apreciar en (7), el estudiante, al ver que no dispone de bolígrafo, se levanta para ir a coger uno en su mochila y lo explicita a la profesora, quien le contesta que tiene uno a disposición. 
(5) $<$ FF015> ¿Tengo que tomar un lápiz o algo asi?

$<$ PROFE $>$ Nada lo tengo todo yo

$<$ FF015 $>$ Vale

(IN_015_FF)

(6) $<$ FF008> ¿Puedo tomar un... un ... bic o no sé?

$<$ PROFE $>$ No, no, no, perdón, estoy un poco despistada

$<$ FF008 $>$ (risas) [ningún problema]

$<$ PROFE $>$ [Aquí tienes] (IN_008_FF)

(7) $<$ FF027> Voy a tomar un ... [un boli]

$<$ PROFE $>$ [¿O quieres] [uno?]

$<$ FF027 $>$ [Ah sí] $<$ PROFE $>$ Aquí tengo uno preparado.

$<$ FF027 $>$ Gracias.

(IN_027_FF)

Frente a resultados tan marcados como los de la Tabla 3, la hipótesis del uso elevado de formulaciones indirectas en español por parte de los estudiantes francófonos se corrobora. La razón por la que tenemos una separación tan acentuada entre los recursos directos e indirectos (no) convencionalizados puede ser por el tipo de situación en la que se encuentran los participantes. Como no saben precisamente lo que les espera al entrar en la sala, tienden a no formular peticiones explícitas, pero, en cambio, las realizan bajo sugerencias. Como no conocen el desarrollo del experimento, no se atreven a destacar lo que la profesora hizo mal, lo que olvidó o lo que falta según ellos. De hecho, la formulación de predilección de numerosos estudiantes frente a una mala copia es: ¿es normal? Esto confirma que funciona la distracción prevista por la profesora (ya que se centran totalmente en la redacción), y que no se esperan a los problemas que surgen respecto a ella. En sí, utilizar estas peticiones muy indirectas ya es una forma de atenuar su discurso. Como explicamos a continuación en el análisis de los demás atenuadores, el hecho de escoger una petición en forma de alusión o de elíptico les permite a los estudiantes comunicarse con vaguedad. La profesora tiene que inferir sus peticiones. Detallamos este punto en la sección siguiente (§ 3.2).

\subsection{Peticiones atenuadas y no atenuadas}

La Tabla 4 ilustra la repartición de peticiones atenuadas en el corpus. De las 102 peticiones hechas en las 7 situaciones preparadas ( $c f$. Figura 2), 25 no son atenuadas. Las demás 77 sí lo son. Como puede haber varios recursos de atenuación para una misma petición, en realidad hay más estrategias de atenuación (84) que peticiones (77).

\begin{tabular}{|l|l|}
\hline Atenuación & Frecuencia \\
\hline No & 25 \\
\hline Sí & 77 \\
\hline Total & $\mathbf{1 0 2}$ \\
\hline
\end{tabular}

Tabla 4. Peticiones atenuadas y no atenuadas.

No obstante, el análisis pone de manifiesto que la mayoría de las estrategias no atenuadas son alusiones, especialmente en el caso de la petición de repetir una frase incomprensible. 
Huelga decir entonces que estas alusiones ya son un recurso de atenuación y que no necesitan otro recurso de atenuación adicional. En (8), la persona $<$ FF022 $>$ dice que hay un bolso en la silla y que habría que hacer algo con ello. Lo hace mediante una petición con una alusión. Aunque este ejemplo es una alusión sin atenuación, cabe precisar que muchas de las ocurrencias de alusiones además se atenúan. El ejemplo (9) representa un tipo de alusión frecuentemente presente en el corpus. Allí, el hecho de añadir la oración subordinada porque no sé leer (sic), es una manera de atenuar la petición, ya que se recurre a una justificación. El estudiante justifica por qué necesita hacer la petición y, además, por qué necesita otra hoja.

(8) $<$ FF022> Oh... ¿Es tuyo?

$<$ PROFE $>$ Oh, sí, perdona. (risas) Gracias.

(IN_022_FF)

(9) $<$ PROFE $>$ OK, te dejo leer las consignas.

$<$ FF020 $>$ Eh, (mostrando la hoja) ¿tiene otra porque no sé leer? (sic) (risas)

$<$ PROFE $>$ ¿Qué es esto? Lo siento, lo siento, eh no, eso nooo, a ver si te doy otra copia.

(IN_020_FF)

En cuanto a las peticiones formuladas con elipsis, esta estrategia abarca perspectivas lingüísticas y pragmáticas divergentes. Efectivamente, una elipsis es, a nivel gramatical, bastante indirecto puesto que no contiene verbo que indicaría la intención y la fuerza ilocutiva del hablante. Con una elipsis, se especifica tan solo el objeto de la petición, la persona a quien se dirige y que tiene que llevar a cabo la petición, o se utiliza una forma gramaticalizada. No obstante, a nivel pragmático, la petición sí es muy directa y en ocasiones, se le puede atribuir entonces un carácter poco cortés. Véase el ejemplo (10), donde el estudiante pide a la profesora que repita la información que acaba de pronunciar. La pregunta ¿Cómo? se analiza, al igual que otros recursos parecidos, como una petición elíptica ya que, con ella, se centra en una sola parte de la frase y se puede analizar como petición tan solo con el contexto. En este caso, la petición no se atenúa y parece bastante directa a nivel pragmático.

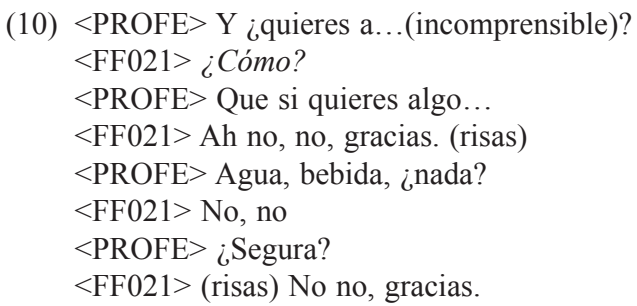
(IN_021_FF)

Otro recurso de atenuación es el hecho de orientar la petición hacia el propio emisor. Este fenómeno está bastante presente en el corpus puesto que corresponde a un $30 \%$ de las ocurrencias ( $c f$. Tabla 3). Como ya explicábamos en $\S 3.1$, el estudiante, en vez de hacer la petición hacia la profesora, se incluye en la petición y la realiza mediante una petición de permiso, una petición/pregunta acerca del desarrollo del experimento o, en varios casos, mediante aserciones. En el apartado $\S 3.3$, examinamos los recursos de atenuación utilizados por los estudiantes francófonos. Analizamos, por una parte, los atenuadores de lo dicho y, por otra, los del decir. 


\subsection{Atenuación de lo dicho y del decir}

Como señalábamos, existen dos tipos de atenuación diferentes: la atenuación de lo dicho y del decir (Briz 2007, Briz y Albelda 2013). Siguiendo a Albelda y Cestero (2011: 15), la atenuación tiene dos funciones principales: reducir el valor significativo de un enunciado (atenuación de lo dicho) o reducir la fuerza ilocutiva (atenuación del decir).

Como se desprende de la Tabla 5, los ejemplos de atenuación del decir son frecuentes en el corpus. Se encuentran no menos de 129 casos, frente a 16 ocurrencias de fenómenos de lo dicho. No cabe duda a este nivel que el tipo de peticiones que tienen que formular los participantes juega un papel preponderante en esta repartición.

\begin{tabular}{|l|l|}
\hline Atenuación & Frecuencia \\
\hline Decir & 129 \\
\hline Dicho & 16 \\
\hline Total & $\mathbf{1 4 5}$ \\
\hline
\end{tabular}

Tabla 5. Repartición de la atenuación de lo dicho y del decir.

En nuestro corpus, la atenuación del decir es el recurso más utilizado para atenuar las peticiones. Este resultado parece obvio, visto el tipo de situaciones analizado. Efectivamente, los estudiantes que piden un bolígrafo para rellenar su formulario no se encuentran en una situación de mucha amenaza. En general, basta con reducir la fuerza ilocutiva de su petición (atenuación del decir) pero no es necesario atenuar el contenido del mensaje (atenuación de lo dicho). Consta decir que, cuando se utiliza una atenuación de lo dicho, se hace en combinación con una atenuación del decir. En algunas ocasiones, no obstante, utilizan la atenuación de lo dicho para no ofender al oyente, y con el fin de dar a conocer que hay un problema, pero sin querer insinuar que la profesora está mal organizada o que lo ha hecho mal. En el ejemplo (11), la atenuación de lo dicho aparece en combinación con la atenuación del decir. Aquí, el estudiante quiere pedir otra hoja ya que la suya no le permite leer correctamente las consignas para llevar a cabo la redacción. Pide una copia de mejor calidad utilizando una petición indirecta (una alusión) y, a nivel de la atenuación, no sólo hay una lítote no muy bien (atenuación de lo dicho) sino también una frase que no veo muy bien, que forma parte de la categoría de la atenuación del decir. Con esta frase -que, siguiendo a Gras (2016), consideramos como una frase principal-, el estudiante da a entender que quiere otra copia. El doble uso de recursos de atenuación se explica por la amenaza que constituye al profesor, ya que sugiere que ha hecho mal su trabajo.

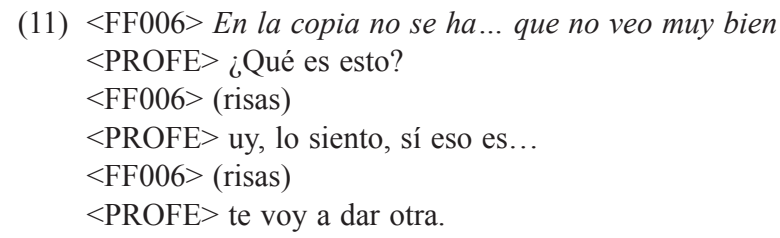


La Tabla 6 da un panorama de las estrategias de atenuación del decir, según las categorías propuestas por Albelda y Cestero (2011). Nos enfocamos en las estrategias más utilizadas, típicamente las que implican al oyente (35 ocurrencias), las que permiten rebajar la aserción de la petición del estudiante (33 ocurrencias), las que corrigen y/o reparan la amenaza que constituye la petición (17 casos) y las que justifican la necesidad de formular una petición (14 casos).

\begin{tabular}{|l|c|}
\hline Decir & $\mathbf{1 2 9}$ \\
\hline acotar/restringir lo dicho & 1 \\
\hline corregir/reparar & 17 \\
\hline desfocalizar elementos de la enunciación & 6 \\
\hline formular acto directivo de forma indirecta & 12 \\
\hline impersonalización & 10 \\
\hline implicar al oyente & 35 \\
\hline justificar & 14 \\
\hline protección imagen & 1 \\
\hline rebajar aserción & 33 \\
\hline
\end{tabular}

Tabla 6. Atenuación del decir.

A continuación, ilustramos estas cuatro estrategias con un ejemplo. En (12), el estudiante usa una petición reorientada hacia sí mismo y, además, emplea una fórmula fática ¿o? que implica al oyente en la petición. Albelda y Cestero (2011: 33) disciernen las fórmulas de petición de consentimiento al tú (¿no?, ¿vale?, ¿qué te parece?) de las de presentación de alternativas, en la que aparece $\dot{i} . . . o$ [qué]? El enunciado ¿o? aparece con relativa frecuencia en nuestro corpus (10 casos) y da la posibilidad al oyente de proponer otra sugerencia. Desde un punto de vista sintáctico, el hablante no termina su frase, lo que constituye otra estrategia para implicar al tú-oyente en lo dicho por el hablante (Albelda y Cestero 2011). En (13), el hablante se centra en el objeto de su petición (el bolígrafo) pero añade una pregunta (¿necesito?) para así rebajar la aserción o para disminuir su manera bastante franca de contestar a la profesora. El ejemplo (14) combina dos estrategias. Primero, introduce su petición con un recurso que hemos considerado como un marcador discursivo (eh), porque tiene el mismo efecto, el de minimizar la disconformidad dialógica y/o atenuar la propia imagen monológicamente. Segundo, también se incluye una justificación después de la formulación del núcleo de la petición (porque no sé leer (sic)). Este mecanismo lleva a "la reducción del peso enunciativo del hablante" (Albelda y Cestero 2011: 28). Aquí, el hablante, al pedir otra copia, siente la necesidad de justificarse y lo hace mediante un gesto (muestra la hoja a la profesora) y una frase. Las risas también constituyen una manera de atenuar la petición.

(12) $<$ FF014> ¿Tengo que hacer un borrador o...?

$<$ PROFE $>$ Eh bueno lo he lo había escrito pero si quieres ya directamente escribirlo [en el papel]

$<$ FF014 $>$ [Sí, creo que sí.]

$<$ PROFE $>$ ¿Sí? Bueno...

(IN_014_FF) 
(13) $<$ PROFE $>$ A ver, te doy un formulario, con la tarea debajo.

$<$ FF003 $>$ Sí.

$<$ PROFE $>$ Es el formulario.

$<$ FF003 $>$ Un boligrafo, ¿necesito?

$<$ PROFE $>$ ¿Cómo?

$<$ FF003 $>$ ¿Un bolígrafo necesito?

$<$ PROFE $>$ Ah sí, te voy a dar uno. Te voy a dar uno. Hop. Aquí tienes.

(IN_003_FF)

(14) $<$ PROFE $>$ OK, te dejo leer las consignas.

$<$ FF020 $>$ Eh, (mostrando la hoja) ¿tiene otra? Porque no sé leer. (risas)

$<$ PROFE $>$ ¿Qué es esto? Lo siento, lo siento, eh no, eso nooo, a ver si

te doy otra copia.

(IN_020_FF)

En la Tabla 7, se ilustran las estrategias de atenuación de lo dicho. Como ya mencionábamos, esta estrategia es mucho menos frecuente que la del decir. La razón de este fenómeno nos parece ser que el estudiante no ve necesario minimizar el contenido de lo que dice, puesto que el contenido de la petición no constituye una amenaza muy grande para su interlocutor.

\begin{tabular}{|ll|l|}
\hline Dicho & & $\mathbf{1 6}$ \\
\hline & expresión de forma vaga & 1 \\
\hline minimizar dicho & 15 \\
\hline
\end{tabular}

Tabla 7. Atenuación de lo dicho.

Un ejemplo típico de esta categoría es (15), donde el estudiante atenúa su discurso mediante solamente. En la misma situación los estudiantes también dicen a menudo que no pueden leer la copia muy bien, o que una parte ha salido mal. Estos cuantificadores y eufemismos permiten disminuir y suavizar la proposición del hablante. Además, como se puede apreciar en este ejemplo, cuando surge una atenuación de lo dicho, se hace en combinación con una atenuación del decir. En (15), se hace mediante una justificación es que no puedo leer (sic).

(15) $<$ PROFE $>$ Entonces se trata de una redacción. Tienes eh 5 diálogos que escribir, bueno te dejo leer las consignas, ¿OK?

$<$ QD $>$ Mh

$<$ PROFE $>$ y si tienes cualquier pregunta, me lo dices, ¿OK?

$<$ QD $>$ Vale euh (risas) solamente es que no puedo leer (risas)

$<$ PROFE $>$ Uy, ¿Qué ha pasado allí? No...

$<$ QD $>$ (incomprensible)

(IN_012_MF)

Las tablas 6 y 7 van ambas en el mismo sentido. Muestran que el estudiante atenúa la fuerza ilocutiva del acto de habla en vez del contenido del mensaje, que sí transmite a la profesora. Aunque estos dos fenómenos, definidos por Albelda y Cestero (2011) como atenuación del decir y atenuación de lo dicho, están íntimamente relacionados, vemos que la distinción se perfila claramente en los datos analizados. 


\section{DISCUSIÓN Y CONCLUSIONES}

El análisis propuesto se ha centrado en la atenuación de las peticiones de hablantes no nativos del español. El marco teórico que hemos utilizado es el de Albelda y Cestero (2011), quienes propusieron una tipología de las diferentes estrategias, tácticas o movimientos generales de la atenuación (2011: 36).

Se corrobora nuestra primera hipótesis acerca de las peticiones que típicamente se suelen realizar y las que se realizan menos. Efectivamente, las peticiones más formuladas se encuentran en situaciones donde el estudiante necesita la intervención de la profesora para avanzar. Pensamos en las peticiones para obtener una copia de mejor calidad, la petición de repetir algo que el estudiante no ha entendido y la petición de un bolígrafo. Las que menos se realizan son las peticiones para beber o comer algo y la de poder consultar un diccionario. Este resultado subraya la importancia de las necesidades y de las expectativas de los estudiantes. Asimismo, los estudiantes añaden peticiones a las peticiones inicialmente previstas por la profesora, pero visto el carácter heterogéneo de estas, no se han tenido en cuenta para este análisis.

La segunda hipótesis, que concierne a un mayor uso de la atenuación en las peticiones más amenazantes para el destinatario, se confirma sólo en parte. En general, los estudiantes suelen atenuar bastante sus peticiones, pero no cuando piden la repetición de una información que no han entendido. Este factor puede ser debido a la rapidez comunicativa. Saben que la profesora espera una respuesta a lo que no han entendido y, para poder darla, utilizan una petición elíptica que les permite formular de manera bastante corta la petición de repetir la frase.

La tercera hipótesis se verifica completamente. En nuestro corpus, encontramos más atenuación del decir que de lo dicho. Además, cuando se utiliza una atenuación de lo dicho, se hace en combinación con una atenuación del decir. La causa de este resultado se debe a que el contenido de la petición no trata de un tema sensible en la interacción profesor-estudiante. Por eso, el estudiante no necesita atenuar demasiado el contenido de su proposición, sólo la forma.

Además de los recursos analizados en este artículo, existen otras tácticas para atenuar el discurso. Por un lado, se pueden atenuar mediante gestos y miradas. Los hemos descartado del presente análisis, pero merecen un estudio detallado en el futuro. El aspecto no verbal puede ser una fuente interesante para apoyar el estudio lingüístico ya que las peticiones también se pueden 'formular' de manera no verbal. Por otro lado, evitar hacer una petición también es una forma de atenuar, ya que el estudiante no quiere amenazar ni la imagen de la profesora ni su propia imagen y escoge no hacer la petición.

A lo largo de nuestro análisis, hemos observado la importancia de las peticiones indirectas no-convencionales. Constituyen en sí una atenuación frente a peticiones menos indirectas. Así, se apuntan muchas peticiones bajo forma de alusiones o peticiones reorientadas hacia el propio emisor. Esta última estrategia consiste en un cambio de perspectiva. El hablante, en vez de pedir al oyente que cumpla la petición, señala que la realiza él mismo o pide permiso para realizarla.

Frente a resultados tan marcados como los del presente análisis, sería útil, por un lado, comparar las respuestas no nativas con las de estudiantes nativos del español y, por otro lado, ver las semejanzas y diferencias con la lengua materna de los estudiantes no nativos. 
Es decir, comparar la formulación de las peticiones en su lengua materna (el francés) y en la lengua extranjera (el español). Este componente está previsto en estudios futuros, además de ampliar el corpus. Esto nos parece imprescindible para apreciar plenamente la calidad de producción de los estudiantes no nativos.

\section{Referencias bibliográficas}

Albelda, M. (2010). “¿Cómo se reconoce la atenuación? Una aproximación metodológica basada en el español peninsular hablado". En Orletti, F. y L. Mariottini (eds.). (Des)cortesía en español, Università Roma Tre, pp. 41-70.

Albelda, M. y A. Briz (2010). "Cortesía y atenuantes verbales en las dos orillas a través de muestras orales”. En Aleza, M. y J.M. Enguita (coords.). La lengua española en América: normas y usos actuales, Universitat de València, pp. 237-260.

Albelda, M. y A. M. ${ }^{a}$ Cestero (2011). "De nuevo, sobre los procedimientos de atenuación lingüística", Español Actual, 96, pp. 121-155.

Alonso Pérez-Ávila, E. (2005). "Peticiones en español. Aproximación a la pragmática de la interlengua”, Artifara, 5: http://www.cisi.unito.it/artifara/rivista5/testi/peticiones.asp (20-03-2017).

Bataller, R. (2010). "Making a Request for a Service in Spanish: Pragmatic Development in the Study Abroad Setting", Foreign Language Annals, 43 (1): 160-175.

Bataller, R. (2013). "Role-plays vs. natural data: asking for a drink at a cafeteria in peninsular Spanish", Íkala, revista de lenguaje y cultura, 18 (2), pp. 111-126.

Blum-Kulka, S. (1987). "Indirectness and Politeness in Requests: Same or Different?", Journal of Pragmatics, 11, pp. 131-146.

Blum-Kulka, S. y J. House (1989). "Cross-cultural and Situational Variation in Requesting Behavior". En Blum-Kulka, S., House, J. y G. Kasper (eds.), Cross-cultural Pragmatics: Requests and Apologies, Norwood: Ablex, pp. 123-54.

Blum-Kulka, S. House, J. y G. Kasper (eds.) (1989). Cross-cultural Pragmatics: Requests and Apologies. Norwood: Ablex.

Briz, A. (1998). El español coloquial en la conversación. Esbozo de pragmagramática. Barcelona: Ariel.

Briz, A. (2007). "Para un análisis semántico, pragmático y sociopragmático de la cortesía atenuadora en España y América", Lingüística Española Actual, XXIX/1, pp. 5-44.

Briz, A. y M. Albelda (2013). "Una propuesta teórica y metodológica para el análisis de la atenuación lingüística en español y portugués. La base de un proyecto en común (ES.POR.ATENUACIÓN)”. Onomázein, 28, pp. 288-319.

Carduner, J. (1998). Politeness strategies of intermediate to advanced learners of Spanish. Unpublished PhD thesis, University of Pittsburgh, PA.

Castillo Lluch, M. (2009). "Étude interculturelle des formes nominales de l'adresse en français et en espagnol contemporains". En Araújo Carreira, M. H. (ed.). Termes d'adresse et modalités énonciatives dans les langues romanes. Saint-Denis: Université Paris 8, pp. 203-214.

CIEL-F. Corpus International Écologique de la Langue Française. (2008-2013): http://www.ciel-f. org/ (21-03-2017).

CORLEC. Corpus del Español centro-peninsular, dirigido por Francisco Marcos Marín en la Universidad Autónoma de Madrid con el apoyo de la Agencia Nacional para el Desarrollo de Programas del V Centenario (1990-1993). http://www.lllf.uam.es/ESP/Corlec.html (20-03-2017).

Escandell Vidal, M. ${ }^{a}$ V. (1996). "Los fenómenos de interferencia pragmática”. En Miquel, L. y N. Sans (eds.), Didáctica del Español como lengua extranjera, Expolingua, pp. 95-109.

Escandell Vidal, M. ${ }^{a}$ V. (2009). "Social Cognition and Second Language Learning". En GómezMorón, R., Padilla Cruz, M., Fernández Amaya, L. y M.O. Hernández López (eds.). Pragmatics Applied to Language Teaching and Learning. Newcastle: Cambridge Scholars, pp. 1-39. 
Gras, P. (2016). "Revisiting the functional typology of insubordination: que-initial sentences in Spanish". En Evans, N. y H. Watanabe (eds.), Dynamics of Insubordination..Amsterdam: John Benjamins, pp. 113-144.

Hassall, T. (2003). "Requests by Australian Learners of Indonesian", Journal of Pragmatics, 35, pp. 1903-1928.

Janney, R. and Arndt, H. (1992). "Intracultural tact versus intercultural tact”. En Watts, R. J., Ide, S. y K. Ehlich (dirs.). Politeness in Language: studies in its History, Theory and Practice. Berlin: Mouton de Gruyter, pp. 21-42.

Marsily, A. (2016): COPINE. Corpus Oral de Peticiones en Interacciones Naturalizadas en Español. Université catholique de Louvain.

Marsily, A. (2018). "Directness vs. indirectness. A contrastive analysis of pragmatic equivalence in Spanish and French request formulations". Languages in Contrast, 18(1), pp. 122-144.

Mir, M. (1993). "Direct requests can also be polite". Paper presented at the 7th Annual Meeting of the International Conference on Pragmatics and Language Learning. University of Illinois: UrbanaChampaign. 1-3 de abril.

Placencia, M. E. (1998). "Pragmatic variation: Ecuadorian Spanish vs. Peninsular Spanish”, Spanish Applied Linguistics, 2 (1), pp. 71-106.

Placencia, M. E. (2005). "Pragmatic Variation in Corner Store Interactions in Quito and Madrid", Hispania, 88(3), pp. 583-598.

Placencia, M. E. y A. Mancera Rueda (2011). “'Dame un cortado de máquina cuando puedas’: estrategias de cortesía en la realización de la trasacción central en bares de Sevilla". En Fuentes Rodríguez, C., Alcaide Lara, E. y E. Brenes Peña (eds.). Aproximaciones a la (des)cortesía verbal en español. Bern: Peter Lang, pp. 487-504.

Tran, G.Q. (2006). "The naturalized role-play: An innovative methodology in cross-cultural and interlanguage pragmatics research", Reflections on English Language Teaching, 5 (2), pp. 1-24.

Valero Aguilar, A. (2008). "Inmersión en la pragmática en el aula". En: Actas del II Congreso Internacional de Lengua, Literatura y Cultura de E/LE: Teoría y práctica docente. Onda: JMC, pp. 363-378.

\section{ANEJOS}

\section{Taxonomía de las peticiones}

\begin{tabular}{|c|c|c|c|}
\hline Directo & $\begin{array}{l}\text { Indirecto } \\
\text { convencional }\end{array}$ & $\begin{array}{l}\text { Indirecto no- } \\
\text { convencional }\end{array}$ & $\begin{array}{l}\text { Reorientación de la } \\
\text { petición desde emisor }\end{array}$ \\
\hline $\begin{array}{l}\text { Modo derivable } \\
\text { - Infinitivo } \\
\text { - Imperativo } \\
\text { - Indicativo } \\
\text { - } Q U E+\text { subjuntivo }\end{array}$ & $\begin{array}{l}\text { Fórmulas de } \\
\text { sugerencia }\end{array}$ & $\begin{array}{l}\text { Alusión } \\
\text { - Pregunta } \\
\text { - Afirmación }\end{array}$ & Permiso \\
\hline $\begin{array}{l}\text { Performativos } \\
\text { - Realizativas } \\
\text { - Casi realizativas }\end{array}$ & $\begin{array}{l}\text { Pregunta preparatoria } \\
\text { - Habilidad } \\
\text { - Voluntad } \\
\text { - Permiso } \\
\text { - Posibilidad }\end{array}$ & $\begin{array}{l}\text { Elíptico } \\
\text { - Vocativo } \\
\text { - Objeto de la } \\
\text { petición } \\
\text { - Partícula } \\
\text { gramaticalizada }\end{array}$ & Pregunta \\
\hline Declaración obligativa & Interrogativo simple & Declaración volitiva & Aserción \\
\hline
\end{tabular}

